\title{
An Investigation of Millimeter Wave Reflectarrays for Small Satellite Platforms
}

\author{
Ghulam Ahmad*1, Tim W.C. Brown ${ }^{1}$, Craig I. Underwood ${ }^{1}$, Tian H. Loh ${ }^{2}$ \\ ${ }^{1}$ Fuculty of Electroncs and Physical Sciences, University of Surrey, Guildford, GU2 7XH \\ United Kingdom \\ ${ }^{2}$ National Physical Laboratory, Teddington, TW11 OLW, United Kingdom
}

\begin{abstract}
This article reports two contributions related to reflectarray antenna design at millimeter waves (mm-waves). First, a closed form analytical formulation is provided for the prediction of reflection properties of square/rectangular mmwaves reflectarray unit cells based on various quality factors and the theory of waveguide coupled resonators. To ensure a high accuracy at mm-waves, the effects of fringing fields, surface waves, metal conductivity, and metal surface roughness are included in the analysis. This analysis program greatly facilitates the parametric studies of a unit cell's constituting parameters to converge on an optimum design solution. Secondly, the concept of phase quantization is proposed for a cost effective realization of mm-waves reflectarrays. The developed formulation in the first contribution was used to design two 3 bit phase quantized, single layer, 19 wavelength, passive reflectarrays at $60 \mathrm{GHz}$. The test results are compared with simulations and a very good agreement was observed. These findings are potentially useful for the realization of high gain antennas for mm-wave inter-satellite links in small satellite platforms.
\end{abstract}

Keywords: Antenna, Reflectarray, Reflection Loss, Satellite, Unit Cell

\footnotetext{
* Corresponding author

Email address: g.ahmad@surrey.ac.uk (Ghulam Ahmad)
} 


\section{Introduction}

Millimeter waves (mm-waves) satellite and terrestrial backhaul communication links require high gain antennas to counter severe propagation losses. Two commonly used high gain solutions at lower frequencies are the reflector 5 antennas, and antenna arrays. Both of these technologies suffer significant disadvantages when applied to mm-waves. Reflector antennas are curved surfaces which are expensive to accurately manufacture at mm-waves. Additionally, the aperture geometry to achieve high gain and a limited accommodation space in the launch fairing may cause conflicting requirements. Similarly, modern antenna arrays are made using printed microstrip technology. These arrays use power distribution network (PDN) to feed the array elements. This PDN has a significant loss at mm-waves which can be as high as $42 \mathrm{~dB}$ as observed by [1, 2]. A high gain antenna array may contain thousands of individual antenna elements [3, 4]. Therefore, the power distribution network becomes very complicated and lossy which drastically affects the antenna performance.

A solution to avoid curved surfaces and lossy power distribution networks is to combine the best features of reflector and array technologies. A reflectarray being an optimal genetic hybrid of these technologies preserves the spatial feeding of reflector technology and the planar structure of printed arrays to achieve high antenna gains [5] Reflectarrays can be stowed along the side panel of a small satellite platform to get accommodated in a limited launch fairing space. To allow further compactness, reflectarrays can be folded into a compact form during launch, and then can be unfolded and deployed once in orbit. To further facilitate the compactness, reflectarrays can be made inflatable 8 10. A reflectarray can implement any reflector antenna configurations to fit the requirements. Due to a flat structure reflectarrays are a potential candidate for aircrafts, and next generation terrestrial backhauls. Moreover, reflectarrays would be potentially useful for high capacity mm-wave inter-satellite links using small satellite platforms. Modern reflectarrays can also benefit from innovative material technologies e.g. phase change materials [11, 12, liquid crystals [13], 
and tunable graphene [14. Reconfiguration devices can be embedded in reflectarray unit cells [15, 16] to to enhance their functionality including contoured beams [17.

A reflectarray spatially illuminates its comprising individual antenna ele35 ments (called unit cells) which reflect the incident electromagnetic (EM) field. The reflected EM field is engineered at each unit cell's location to artificially imitate the parabolic effect. In a well designed reflectarray the performance of unit cells directly impacts the overall performance of a reflectarray [18 20].

The reflection response (magnitude and phase) of a unit cell is the primary determinant of its behavior over a frequency range. For a microstrip based unit cell the reflection response is determined by the substrate dielectric constant $\left(\epsilon_{r}\right)$, loss tangent $(\tan \delta)$, substrate thickness $(h)$, metal conductivity $(\sigma)$, and the reflecting surface geometry. Due to multiple variables, there may exist multiple combinations leading to the same resonant frequency but completely different reflection properties. To synthesize an optimum design one should make best use of the available degrees of freedom to achieve the desired performance. Therefore, it is critical to completely characterize and understand the properties of a unit cell in relation to its parametric variables to avoid accidental misbehavior in the reflection response [21].

Until recently, most of the unit cell designs mainly relied on full wave electromagnetic (FW-EM) simulations. Widely used simulation packages including CST Microwave Studio [22 26] and HFSS [27 29] have been used to design and characterize the unit cells. Due to multiple degrees of freedom in a design; to reach an optimum design, a designer has to bear heavy simulation loads in terms of optimizations and parameter sweeps where each set of parameters costs a separate FW-EM simulation. This process of heavy simulations can be avoided by a first hand parameterized analytical tool for the unit cell performance prediction. From the results of this mathematical model, the designer can choose unit cell design parameters followed by only a single or few EM simulations to 60 converge on the optimum design thereby significantly reducing the design efforts. Although, this analytical technique do not replace the need for FW-EM 
simulations, however it significantly reduces the number of such simulations.

A unit cell can be represented by an equivalent $R L C$ resonator. The modeling of a waveguide coupled resonator under the assumption of small perturbations to approximate the energy decay in a resonator is presented in this paper. Theory developed in [30] for the prediction of reflection properties of a resonator in terms of various quality factors (Q) is extended to apply to a reflectarray unit cell and the effects of fringing fields, surface waves, metal conductivity, and metal surface roughness are included in the model to make it valid at mm-waves. In a reflectarray design, usually a continuous reflection phase range is required from unit cells 5 . However, in practice it is not feasible to make such a continuous reflection phase available particularly at higher frequencies. Therefore, we have proposed the concept of phase quantization in reflectarrays. Two 3 bit phase quantized reflectarrays were designed based on

75 the results from the unit cell mathematical model. The measured performance of these reflectarrays is compared with their FW-EM simulated results where a strong agreement was observed.

\section{Reflectarray operation and unit cell design}

This section presents the reflectarray operational concept, and the analysis of microstrip printed unit cells for mm-waves operation.

\subsection{Reflectarray geometry and operation}

The geometry of a basic reflectarray [16, 31, 32 is shown in Fig. 1. Reflectarrays have mainly planar reflecting surfaces, although they can also be made slightly curved. In its basic form, a reflectarray surface is designed with an objective to collimate the scattered field in its far field. The number of elements/unit cells in a reflectarray is determined by the required gain. Usually, a reflectarray is composed of thousands of unit cells. A feed source antenna having its phase center coinciding with the array focal point $(0,0, F)$ spatially illuminates these unit cells. The radiated field from the feed horn is scattered 


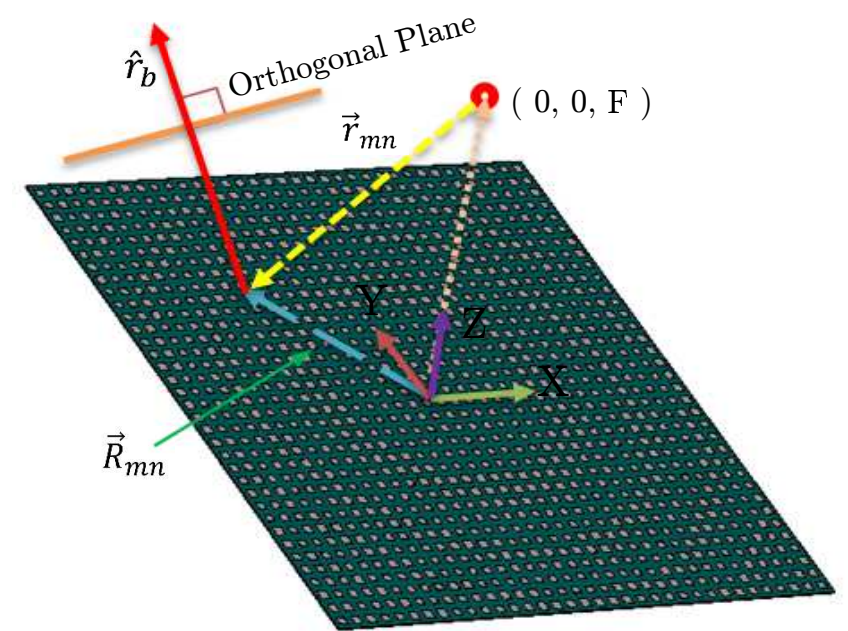

Figure 1: Printed reflectarray antenna on a grounded substrate

by these unit cells. At the location of each unit cell a phase correction is applied to the scattered field. Therefore, the parabola effect is artificially engineered by scattered field phase correction from the entire reflectarray surface. The phase of the reflected field in a plane orthogonal to the direction of radiated beam is constant [16] as follows:

$$
k_{\mathrm{o}}\left(r_{m n}-\vec{R}_{m n} \cdot \hat{r}_{b}\right)-\triangle \phi_{m n}=2 \pi N
$$

where $k_{\mathrm{o}}=2 \pi / \lambda$ is the free space wave number for wavelength $\lambda, \vec{r}_{m n}$ is the position vector of $m n^{\text {th }}$ unit cell w.r.t. array focal point $(0,0, F), \vec{R}_{m n}$ is the position vector of $m n^{\text {th }}$ unit cell relative to the array center/origin of coordinate ${ }_{85}$ system $(0,0,0), \hat{r}_{b}$ is the direction of radiated pencil beam, F represents array focal length, and $N$ is an integer. Each $m n^{\text {th }}$ unit cell introduces a phase shift equal to $\triangle \phi_{m n}$ between the incident and scattered field. By controlling this localized phase at each unit cell location, the pencil beam can be scanned or a contoured beam can be synthesized. As a reflectarray operates on the 90 principle of reflecting the incident field on unit cells, it is required to minimize the reflection loss occurring in unit cells. In ideal conditions the magnitude of the phase corrected reflected field from unit cells should be equal to the magnitude of the incident field. However, due to losses in a substrate and a 
finite conductivity of reflecting metal surface, the reflected field suffers certain amount of loss which one would try to minimize in a design. In a well-designed reflectarray, it is mainly the unit cell behavior which determines the overall characteristics of a reflectarray. Therefore, significant design efforts are focused to develop an optimum unit cell.

\subsection{Unit cell analytics}

A reflectarray unit cell is shown in Fig. 2. It consists of a reflecting geometry (square patch here) on a grounded substrate. The reflecting geometry of a patch can be a simple regular shape or a compound shape resulting from a combination of basic shapes. As the frequency increases the fabrication tolerances become more severe. The fractional change in geometry due to these tolerances is significantly higher at mm-waves in comparison to low frequencies. Although, one may be tempted to use compound reflecting shapes at mm-waves, it is potentially difficult to accurately fabricate at these frequencies using low cost fabrication process providing a tolerance in the range of $100-200 \mu \mathrm{m}$. As the reflection response of a unit cell is a complex function of its parameters, a change in the compound shape's geometry may cause drastic variations from its design with no guarantee of the required performance. A complex radiating shape is not a suitable solution at mm-waves. Therefore, very basic shapes including rectangular, square, and circles are preferred at mm-waves. When these basic shapes are subject to fabrication tolerances, the resulting geometries are still expected to be able to achieve the required performance at a slightly different frequency. Similarly, simple shapes result in closed form analytical solutions. Analysis presented here is applicable to rectangular and square reflecting patches which are envisioned to be widely used at mm-waves. It can be extended to other shapes provided the suitable expressions for radiated power can be realized.

It is well known that the unit cell reflection properties are measured using a waveguide setup as it provides periodic boundary conditions required for the unit cell 33. Such a structure inherently models mutual coupling of elements in an infinite array environment. The reflection phase of a unit cell is a func- 
tion of the wave indecent angle. In a well designed reflectarray, the incident angle from feed horn for the edge located unit cells in an actual reflectarray is nearly equal to the waveguide incidence angle at a unit cell. Usually, the phase variations expected due to the violation of local periodicity in an actual reflectarray are less than the phase variations due to the wave incident angle. Therefore, a waveguide test setup is considered as a reliable source for the unit cell measurements. A reflectarray unit cell can be represented by an equivalent $R L C$ circuit resonator. The waveguide coupled resonator theory was developed in 30] based on the theory of small perturbations [34. A unit cell's equivalent $R L C$ resonator coupled to a dominant mode metallic waveguide is shown in Fig. 3. The reflection coefficient of a unit cell under this scenario is given as:

$$
\Gamma=\frac{\frac{1}{Q_{\mathrm{ext}}}-\frac{1}{Q_{\mathrm{o}}}-\frac{2 \mathrm{j}\left(f-f_{\mathrm{o}}\right)}{f_{\mathrm{o}}}}{\frac{1}{Q_{\mathrm{ext}}}+\frac{1}{Q_{\mathrm{o}}}+\frac{2 \mathrm{j}\left(f-f_{\mathrm{o}}\right)}{f_{\mathrm{o}}}}
$$

where $\Gamma$ is the reflection coefficient of the unit cell resonator, $f$ is the frequency of interest around the resonant frequency $f_{\mathrm{o}}, Q_{\mathrm{o}}$ is the quality factor to account for the conductor and dielectric losses, and $Q_{\text {ext }}$ is the quality factor which accounts for radiations from the unit cell including effects of surface waves. $Q_{\mathrm{o}}$ is given as:

$$
\frac{1}{Q_{\mathrm{o}}}=\frac{1}{Q_{\mathrm{d}}}+\frac{1}{Q_{\mathrm{c}}}
$$

where, the quality factor $Q_{\mathrm{d}}$ relating to dielectric loss due to $\tan \delta$ of the mi-
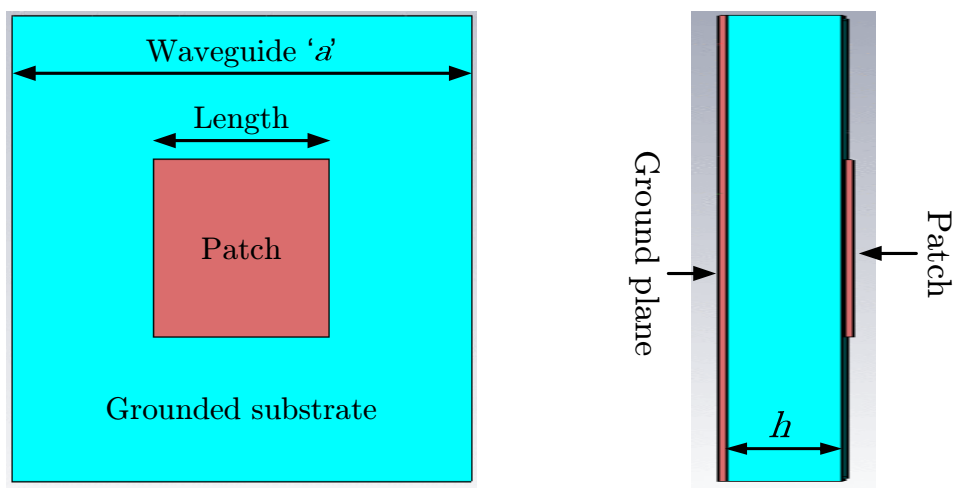

Figure 2: A reflectarray unit cell (front and side views). 


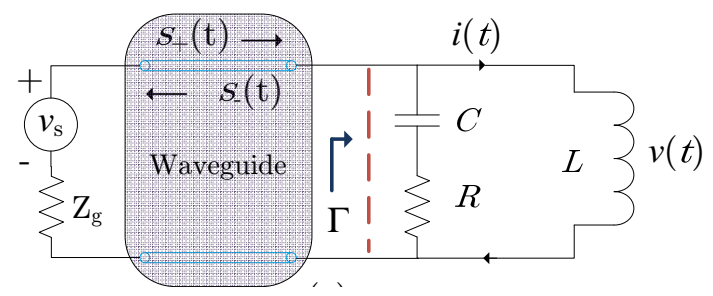

Figure 3: A unit cell resonator coupled to the waveguide with an external excitation source (dominant mode waveguide incidence). The incident wave is represented by $s_{+}(t)$, the reflected wave is represented by $s_{-}(t)$ and the reflection coefficient of the unit cell is represented by $\Gamma$.

crostrip substrate is defined by [35], and $Q_{\mathrm{c}}$ is the quality factor due to the finite conductivity of a rough metal reflecting surface of the unit cell. The surface roughness is mainly ignored at lower frequencies. At mm-wave frequencies, surface roughness becomes very comparable to the skin depth, thereby increasing the internal losses in a unit cell. The metal surface roughness $R_{\text {rms }}$ ranges from $0.3 \mu \mathrm{m}$ to $2.4 \mu \mathrm{m}$ for commercially available copper depositing techniques used in manufacturing of PCBs [36]. Considering the conductivity of copper, one finds skin depth $\delta_{s}$ of the order $2 \mu \mathrm{m} \leq \delta_{s} \leq 0.2 \mu \mathrm{m}$ in the frequency range of $1 \mathrm{GHz} \leq f \leq 100 \mathrm{GHz}$. It is comparable to the metal surface roughness of commercially available PCB substrates. With an increase in the frequency the dielectric loss tangent increases. Similar is true for the conductor surface roughness. At mm-waves, in most of the cases, loss due to the conductor is higher than the loss due to loss tangent of a wisely chosen substrate. Therefore, it is essential to consider the effects of surface roughness in mm-wave designs. The effective conductivity of a rough surface $\sigma_{r}$ is modeled as [37, 38]:

$$
\sigma_{r}=\frac{\sigma}{\left[1+\frac{2}{\pi} \tan ^{-1}\left\{1.4\left(\frac{R_{\mathrm{rms}}}{\delta_{s}}\right)^{2}\right\}\right]^{2}}
$$

where $\sigma$ is the smooth selected metal conductivity (mostly copper). For thin microstrip substrates $h \ll \lambda_{\mathrm{o}}$ (where $\lambda_{\mathrm{o}}=$ free space wavelength), one can find the rough conductor model based quality factor as: $Q_{\mathrm{c}}=h \sqrt{\pi \mu f_{\mathrm{o}} \sigma_{r}}$ which is particularly important in relation to higher frequencies in the range of $\mathrm{mm}$ - 
waves (here $\mu=$ permeability). The accuracy of this model can be improved even further at higher frequencies by using complex models for the effective metal conductivity.

External quality factor is given as: $Q_{\text {ext }}=2 \pi f_{\mathrm{o}} \eta_{\mathrm{rad}} W_{\mathrm{s}} / P_{\mathrm{rad}}$, where $\eta_{\text {rad }}$ is the radiated wave efficiency defined by [39], $W_{\mathrm{s}}$ is the total stored energy under the radiating surface (patch), and $P_{\text {rad }}$ is the radiated power from the radiating surface into the metallic waveguide having dimensions $a \times b$. For thicker substrates surface waves take a significant amount of power therefore, the value of $\eta_{\mathrm{rad}}$ is lowered as shown in [40]. The $W_{\mathrm{s}}$ and $P_{\mathrm{rad}}$ are derived using cavity model of the patch antenna. At resonance the stored electric and magnetic energies under the patch are equal, therefore $W_{\mathrm{s}}$ can be expressed by $\sqrt{5}$ in relation to the electric field distribution $\left(\vec{E}_{\text {cavity }}\right)$ under the patch for cavity dominant mode i.e. $\mathrm{TM}_{010}$ [4]. Similarly, $P_{\mathrm{rad}}$ is expressed by 6 for TE mode inside the waveguide using its electric $\left(\vec{E}_{\mathrm{WG}}\right)$ and magnetic $\left(\vec{H}_{\mathrm{WG}}\right)$ fields, which in turn is related to the TE mode amplitude $\left(\left|A_{\mathrm{WG}}\right|\right)$ [34] due to the magnetic currents at the radiating edges of the patch on a grounded substrate. Here, $Z_{\mathrm{WG}}$ is the waveguide impedance for $\mathrm{TE}_{10}$ mode.

$$
\begin{gathered}
W_{\mathrm{s}}=2 W_{\mathrm{e}}=\frac{\epsilon}{2} \int\left|\vec{E}_{\text {cavity }}\right|^{2} d v \\
P_{\mathrm{rad}}=\frac{1}{2} \int \vec{E}_{\mathrm{WG}} \times \vec{H}_{\mathrm{WG}} \cdot \overrightarrow{d s}=\frac{\left|A_{\mathrm{WG}}\right|^{2}}{2 Z_{\mathrm{WG}}}
\end{gathered}
$$

For a rectangular/square patch inside a dominant mode metallic waveguide, the expression for $Q_{\text {ext }}$; by using the coupling of a unit cell resonator and waveguide modes, simplifies to (7).

$$
Q_{\mathrm{ext}}=\eta_{\mathrm{rad}} \frac{f_{\mathrm{o}} \pi^{3} \epsilon}{32 h} \frac{b}{a} \frac{W_{\mathrm{eff}} L_{\mathrm{eff}}}{\sin ^{2}\left(\frac{\pi W_{\mathrm{eff}}}{2 a}\right)} \frac{\omega \mu}{\sqrt{\omega^{2} \mu \varepsilon-\left(\frac{\pi}{a}\right)^{2}}}
$$

where $W_{\text {eff }}$ [42, 43] and $L_{\text {eff }}$ 41] are the effective width and length of the radiating patch with physical width $=W$ and length $=L$ respectively, as:

$$
W_{\mathrm{eff}}=\frac{\lambda_{\mathrm{o}}}{2} \sqrt{\frac{2}{\epsilon_{\mathrm{r}}+1}}+2 \Delta W=W+2 \Delta W, \quad L_{\mathrm{eff}}=\frac{c}{2 f \sqrt{\epsilon_{\mathrm{eff}}}}=L+2 \Delta L
$$


where $\Delta W$ and $\Delta L$ are the effective increase in width and length due to fringing fields as defined by [44] and [45] respectively, $\epsilon_{\mathrm{r}}$ is the substrate dielectric constant, $\epsilon_{\mathrm{eff}}$ is the effective dielectric constant for the substrate at the operating frequency as defined by [46]. For more details on coupling of modes one can refer to [34]. The expression for $Q_{\text {ext }}$ accounts for the mutual coupling of unit cells in an infinite array environment due to periodic boundary conditions offered by the metallic waveguide structure.

At resonance, when $Q_{\text {ext }}<Q_{\mathrm{o}}$, is called over coupled condition and $\Gamma\left(f_{\mathrm{o}}\right)=$ $\left|\Gamma\left(f_{\mathrm{o}}\right)\right| e^{j 0}$. This is the only useful condition in a reflectarray design. Therefore, for a reliable operation, the reflectarray unit cells are designed for the over coupled condition. The reflection phase of a unit cell resonator can be derived by taking the argument of 22 as $\Phi_{\text {res }}\left(f, f_{\mathrm{o}}\right)=\arg \left(\Gamma\left(f, f_{\mathrm{o}}\right)\right)$ :

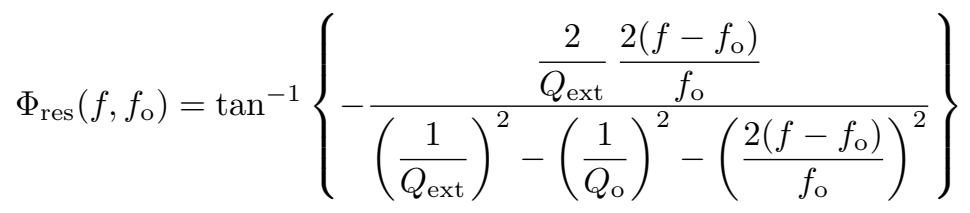

In a variable length phase control technique (envisioned to be widely implemented in mm-wave reflectarrays due to its simplicity), if $L_{\mathrm{o}}$ is the resonant length at center frequency $f_{\mathrm{o}}$, then lengths $L_{\mathrm{o}}+\delta L_{\mathrm{o}}$ and $L_{\mathrm{o}}-\delta L_{\mathrm{o}}$ would corresponds to frequencies $f_{1}$ and $f_{2}$ respectively as in 10 such that $f_{1}<f_{\mathrm{o}}<f_{2}$.

$$
f_{1} \times\left(L_{\mathrm{o}}+\delta L_{\mathrm{o}}\right)=f_{\mathrm{o}} \times L_{\mathrm{o}}=f_{2} \times\left(L_{\mathrm{o}}-\delta L_{\mathrm{o}}\right)
$$

Here, $\delta L_{\mathrm{o}}$ represents a differential change in the length. Therefore, these lengths would correspond to reflection phases as:

$$
\Phi_{1}=\Phi_{\mathrm{res}}\left(f_{\mathrm{o}}, f_{1}\right), \quad \Phi_{2}=\Phi_{\mathrm{res}}\left(f_{\mathrm{o}}, f_{2}\right)
$$

By selecting suitable lengths the required reflection phases from the unit cells can be synthesized. 


\section{Unit cell results and discussions}

This section covers two important results generated through analysis, and simulations. The first outcome is the substrate selection for reflectarray unit cells through analysis. For an optimally selected substrate, the analyitcal results are then compared with the CST Microwave Studio FW-EM simulations as the second outcome.

\subsection{Substrate selection}

Two main considerations while selecting a substrate for reflectarray unit cells are; surface waves generation and the reflection performance. For thin substrates the surface wave effect is negligible. However, for thick substrates its effect needs to be included as illustrated by [47, 48]. At mm-waves $h / \lambda_{\mathrm{o}}$ becomes relatively greater as compared to lower frequencies for the same substrate thickness $(h)$. Therefore, the surface wave effect is more pronounced at mm-wave frequencies. Surface waves are not a strong function of the reflecting element geometrical shape and are mainly characterized by substrate parameters [39, 43. Fig. 4 (a) presents the radiated wave efficiency $\left(\eta_{\mathrm{rad}}\right)$ for various values of the substrate thicknesses and dielectric constants (for more details on $\eta_{\text {rad }}$ one can refer to [40]). It can be observed that for a fixed value of the substrate thickness, a lower dielectric constant leads to a better radiated wave efficiency. Similarly, for a fixed value of dielectric constant a thinner substrate results in a better radiated wave efficiency. To reduce the effect of surface waves generation a thin substrate with a lower value of dielectric constant is preferred.

Fig. 4 (b) displays the reflection coefficienct's magnitude against various substrate thickness values for RO5880 having $\epsilon_{\mathrm{r}}$ of 2.24 . The results considering various effects i.e. metal surface roughness excluded (smooth), included (rough), fringing fields effect (fring), surface waves (SW) are shown. It can be observed that for a suitably selected value of $\epsilon_{\mathrm{r}}$ the effects of surfaces waves are negligible. For substrate thickness values less than 2 mil, a unit cell on this substrate at $60 \mathrm{GHz}$ would behave anomalously. This includes phase misbehavior (a phenomenon due to which the reflection phase performance is severely 


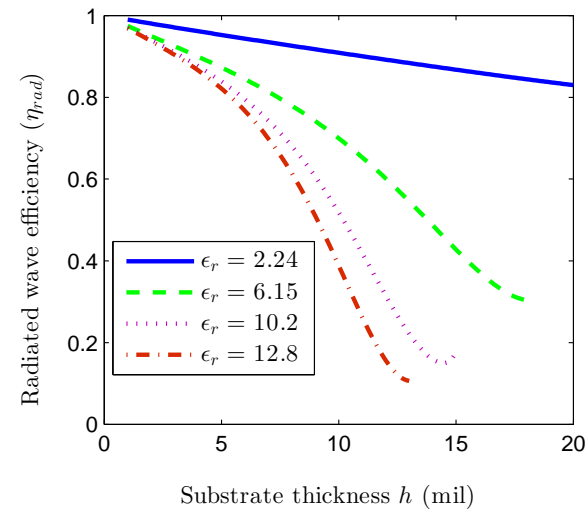

(a) Radiated wave efficiency $\eta_{\text {rad }}$.

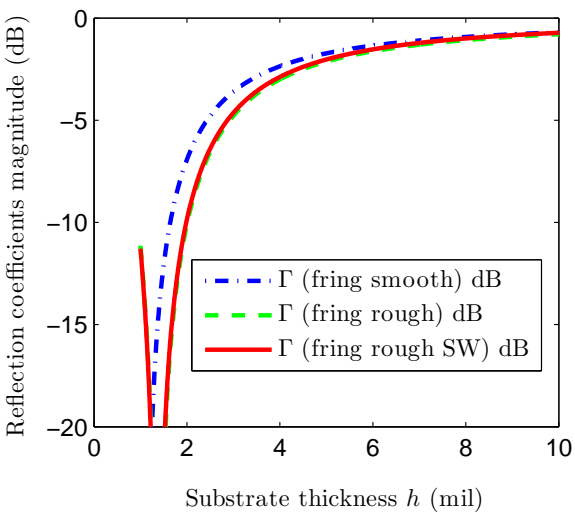

(b) Reflection coefficient's $(\Gamma)$ magnitude.

Figure 4: (a) Radiated wave efficiency $=\eta_{\mathrm{rad}}$ as a function of the substrate thickness for various values of relative dielectric constants. For each $\epsilon_{r}$ case the substrate thickness is limited to allow only the first order mode of surface waves. (b) Reflection coefficient's magnitude as a function of the substrate thickness at $60 \mathrm{GHz}$. $\Gamma$ (fring smooth) considers the effect of fringing fields in microstrip and copper conductivity without its surface roughness. In $\Gamma$ (fring rough) the effect of conductor surface roughness is also considered in addition to fringing fields. While in $\Gamma$ (fring rough SW) the effects of fringing fields, conductor roughness and surface waves are considered.

deteriorated) 21 and/or excessive loss. In either of these cases, the unit cell is not useful in a reflectarray. The phase anomaly and excessive loss issues dictate using a thicker substrate for a reliable operation of the reflectarray.

In Fig. 5 the reflection magnitude and reflection phase of unit cells are plotted for three substrate thickness values of RO5880 with $\epsilon_{\mathrm{r}}$ of 2.24 . For each thickness value the unit cell was designed to resonate at $60 \mathrm{GHz}$. The effects of fringing fields, metal surface roughness and surface waves are introduced iteratively. Due to a suitable choice of $\epsilon_{\mathrm{r}}$ the surface wave effect is not huge. However, it is significant when $\epsilon_{\mathrm{r}}$ is not chosen judiciously as was shown by authors in [40]. It appears from Fig. 5 (a) that the surface waves are playing a positive role in improving the reflection coefficient's magnitude. However, one should realize that these are the results of an individual unit cell inside a metallic waveguide which results in a constructive effect. This can be exploited 
in a reflectarray design in case the surface waves are made to be confined to the

unit cell area only (a solution for this is beyond the scope of the current paper). Here the important point to note is that for an optimal substrate selection in terms of thickness and $\epsilon_{\mathrm{r}}$, the effect of surface waves (which is in general a loss at reflectarray level) and the loss in reflection coefficient's magnitude due to substrate thickness should be minimal. Choosing a too thick substrate would also increase the mass of a reflectarray which can be a concern in small satellites. The reflection phase response in Fig. 5 (b) for three substrate thickness values shown includes the effects of fringing, metal surface roughness and surface waves. There was hardly any significant difference observed in phase response among these cases, therefore only results including all of these effects are plotted. Here, it can be observed that a thicker substrate reduces the achievable reflection phase range from a unit cell. Therefore thicker substrates are required to be avoided for a greater phase swing. Based on this analysis we chose 10 mil thick RO5880 substrate.

It is to note, the time taken by one iteration of the above calculations for a set of given parameters was only $96 \mu$ s on a standard Dell Optiplex desktop machine. Here we explained the effect of substrate thickness and its dielectric constant. One can parameterize any constituting variable of above equations to study its effect on the resulting reflection performance of a unit cell.

\subsection{Comparison of unit cell results}

This section compares the results of analytical analysis with CST Microwave Studio FW-EM simulations of a square unit cell on a 10 mil thick RO5880 substrate using a WR15 square waveguide with aperture dimensions ' $a \times a^{\prime}$. During this analysis the effects of fringing fields, surface waves, and metal surface roughness with finite conductivity were considered. In CST simulations, the surface roughness was input as impedance surface. Fig. 6 displays the reflection performance of a unit cell. Here analytical results produced through the proposed analytical technique are compared with CST simuations. The resonant frequency offset was removed to make the comparison more visible. It can 


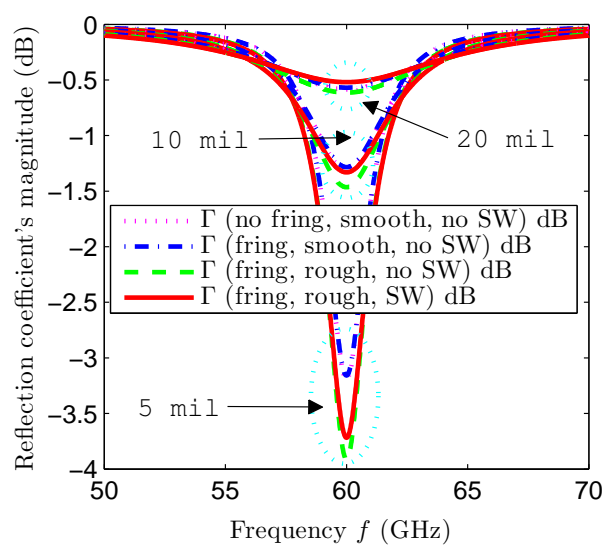

(a) Reflection coefficient's $(\Gamma)$ magnitude.

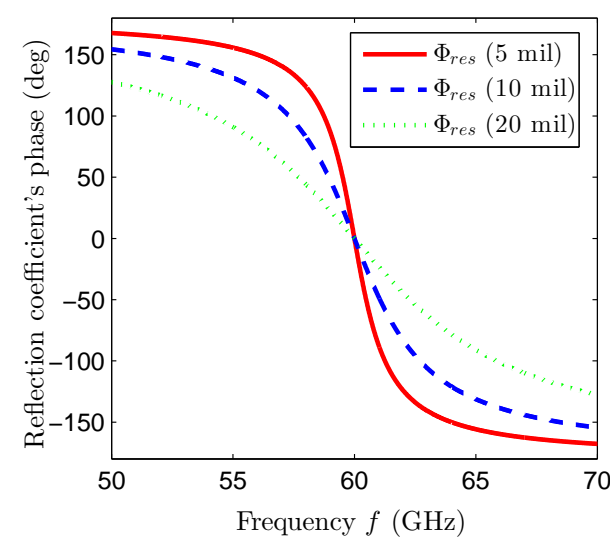

(b) Reflection coefficient's $(\Gamma)$ phase.

Figure 5: Reflection coefficient $(\Gamma)$ versus frequency for various substrate thickness values considering the effects of fringing fields, conductor roughness, and surface waves for a 3.76 mm square unit cell lattice.

be observed that analytical results match very well with the CST simulations. A slightly higher predicted loss in analytical results in comparison to CST results is due to different resulting resonant frequencies in both analyses for a fixed length of the reflecting patch.

Fig. 7 displays the reflection performance versus length for analytical analysis and CST simulations at $60 \mathrm{GHz}$. Here the patch length offset from CST simulation was removed to show the results together. The analytically predicted reflection magnitude and phase responses match very well with the CST simulations. The reflection phase versus length graph is particularly useful when selecting the required patch lengths for the reflectarray design.

In Fig. 8, the offsets are plotted in frequency for a fixed length and in length for a $60 \mathrm{GHz}$ resonance (fixed frequency). There was a frequency offset of about 1.3 GHz which corresponded to a length offset of $100 \mu \mathrm{m}$ between the analytical predictions and CST simulated results which is not huge considering the 60 $\mathrm{GHz}$ operation. This is to note that the analytical technique is based on the theory of small perturbations which do not correct the frequency [30, however predicts the reflection loss accurately. Although, there is a small frequency 


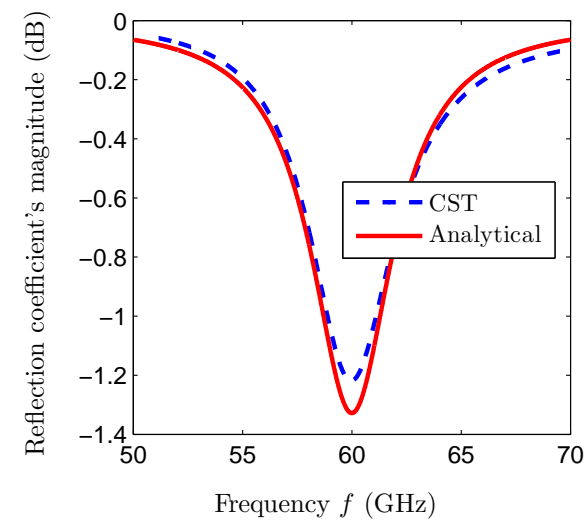

(a) Reflection coefficient's $(\Gamma)$ magnitude.

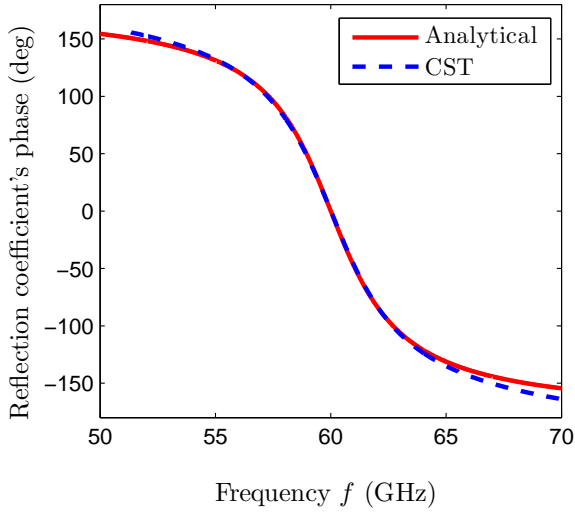

(b) Reflection coefficient's $(\Gamma)$ phase.

Figure 6: A comparison of analytical and CST simulated results of reflection coefficient $(\Gamma)$ versus frequency for 10 mil thick RO5880 substrate considering the effects of fringing fields, conductor roughness, and surface waves for a $3.76 \mathrm{~mm}$ square unit cell lattice.

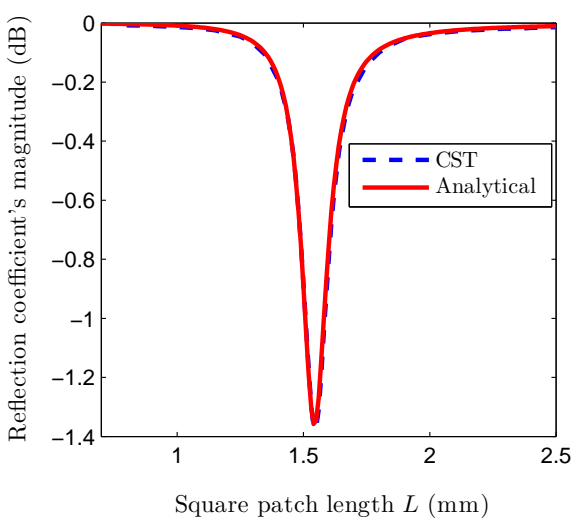

(a) Reflection coefficient's $(\Gamma)$ magnitude.

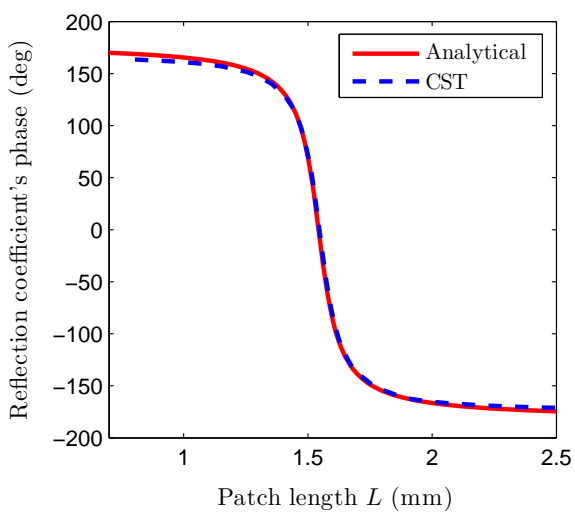

(b) Reflection coefficient's $(\Gamma)$ phase.

Figure 7: A comparison of analytical and CST simulated results of reflection coefficient $(\Gamma)$ versus square patch length for 10 mil thick RO5880 substrate considering the effects of fringing fields, conductor roughness, and surface waves for a $3.76 \mathrm{~mm}$ square unit cell lattice.

offset in analytical predictions, however this is a great first hand analytical tool to perform parametric analysis for an initial design selection followed by only a fewer FW-EM simulations. 


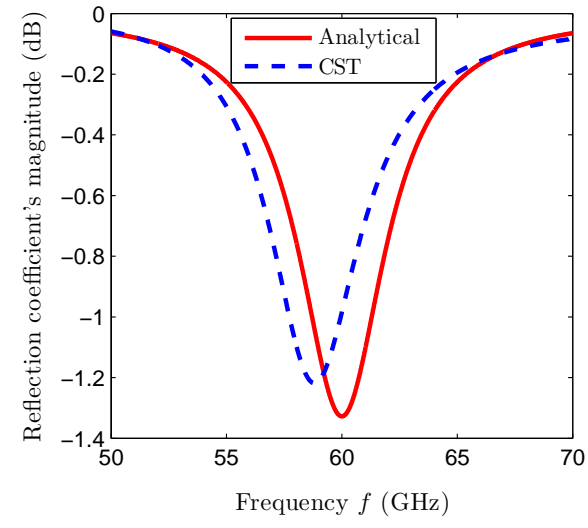

(a) Reflection coefficient's $(\Gamma)$ magnitude.

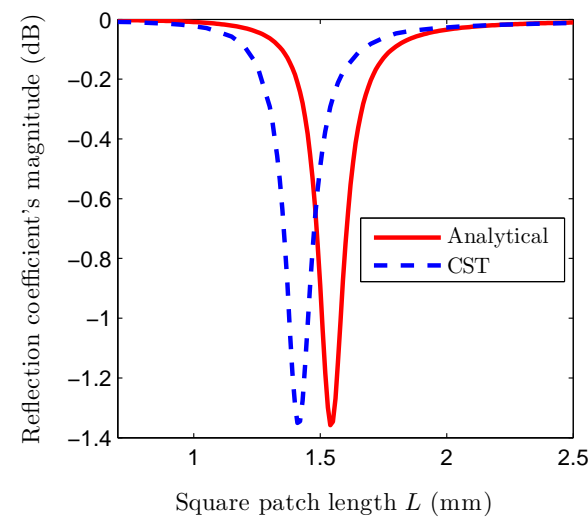

(b) Reflection coefficient's $(\Gamma)$ magnitude.

Figure 8: A comparison of analytical and CST simulated results of reflection coefficient $(\Gamma)$ versus frequency and length variations for a square patch on 10 mil thick RO5880 substrate considering the effects of fringing fields, conductor roughness, and surface waves for a 3.76 mm square unit cell lattice.

\section{Phase quantized reflectarray design}

Simple unit cell shapes do not provide full $360^{\circ}$ phase shift. To alleviate this problem, compound shapes were invented for use at lower frequency bands. However, at mm-waves to ease the fabrication process one need to use simple shapes. A continuous phase shift in a reflectarray design is preferred theoretically. However, when a full $360^{\circ}$ phase range is not available, the performance gets slightly degraded. Further, to implement a continuous phase, one needs to achieve a continuous change in the selected parameter of a unit cell producing this change. This continuous change in unit cell parameters is not feasible in most of the cases. Therefore, it is preferred to implement a discrete set of selected phases which results in a corresponding set of limited unit cells. As it was shown in [49, using a 3 bit phase quantization results in $0.2 \mathrm{~dB}$ reduction in the directivity. Therefore, with a practically acceptable loss in directivity, the implementation problem of passive reflectarrays at mm-waves gets resolved. It is worth noting here that due to the presence of a large number of unit cells, a phase quantized reflectarray can still achieve a comparable performance to 

angles.

We used (1) to calculate the continuous phases required in the reflectarray design. Then these continuous phases were discretized in (12) using the specified quantization phase levels. This discretization results in a 3 bit implementation using a set of 8 unit cells to produce the corresponding 8 phase states.

$$
\Delta \Phi_{Q}=\left\{\begin{array}{lll}
0, & -\frac{9 \pi}{32} \leq\left(\Delta \Phi_{C} \% 2 \pi\right)<\frac{9 \pi}{32} & \text { State 1 } \\
\frac{\pi}{4}, & \frac{9 \pi}{32} \leq\left(\Delta \Phi_{C} \% 2 \pi\right)<\frac{3 \pi}{8} & \text { State 2 } \\
\frac{\pi}{2}, & \frac{3 \pi}{8} \leq\left(\Delta \Phi_{C} \% 2 \pi\right)<\frac{5 \pi}{8} & \text { State 3 } \\
\frac{3 \pi}{4}, & \frac{5 \pi}{8} \leq\left(\Delta \Phi_{C} \% 2 \pi\right)<\frac{7 \pi}{8} & \text { State 4 } \\
\frac{5 \pi}{4}, & \frac{7 \pi}{8} \leq\left(\Delta \Phi_{C} \% 2 \pi\right)<\frac{9 \pi}{8} & \text { State } 5 \\
\frac{3 \pi}{2}, & \frac{11 \pi}{8} \leq\left(\Delta \Phi_{C} \% 2 \pi\right)<\frac{11 \pi}{8} & \text { State 6 } \\
\frac{7 \pi}{4}, & \frac{13 \pi}{8} \leq\left(\Delta \Phi_{C} \% 2 \pi\right)<\frac{13 \pi}{8} & \text { State } 7
\end{array}\right.
$$

Here $\Delta \Phi_{Q}$ is the discrete quantized phase shift introduced by a unit cell, $\Delta \Phi_{C}$ is the desired continuous phase from that particular unit cell as calculated using (1), and \% represents the modulo (remainder) operator. In our variable length implementation to produce the required phase shift from a unit cell, a corresponding set of 8 lengths was selected using (9) to (11).

The unit cells of two 3 bit phase quantized reflectarrays were designed based on the phase quantization scheme presented in section 2.2 using the variable geometry approach on a single layer grounded substrate. One of these was 255 pointing at boresight while the other was designed to point its main beam at $55^{\circ}$. Various parameters for unit cells and reflectarrays are listed in Table 1. A set of 8 unit cells was selected to produce the required quantized reflection phase states in each reflectarray design. Both phase quantized reflectarrays were simulated in CST microwave studio to access their performance. Fig. 9 
Table 1: Parameters of unit cells and reflectarrays.

\begin{tabular}{|l|l|}
\hline Parameter & Value/Description \\
\hline Substrate and thickness $(h)$ & 10 mil thick RO5880 \\
\hline Dielectric constant $\left(\epsilon_{r}\right)$ & 2.24 \\
\hline Loss tangent $(\tan \delta)$ & 0.004 \\
\hline $\begin{array}{l}\text { Copper thickness }(t), \text { and copper sur- } \\
\text { face roughness }\left(R_{\text {rms }}\right) \text { for RO5880 }\end{array}$ & $t=17 \mu \mathrm{m}, R_{\text {rms }}=0.3 \mu \mathrm{m}$ \\
\hline Reflectarray phase quantization & 3 bit $=8$ phase states \\
\hline $\begin{array}{l}\text { Reflectarray focal length }(F), \text { side } \\
\text { length (length }=\text { width, } L=W)\end{array}$ & $F=70 \mathrm{~mm}, L=W=94.5 \mathrm{~mm}$ \\
\hline Inter-element spacing & $2.7 \mathrm{~mm}$ \\
\hline Number of unit cells in the reflectarray & $35 \times 35$ \\
\hline Reflectarrays' pointing angles & $0^{\circ}$ and $55^{\circ}$ \\
\hline Feed horn model & LB-15-10-A from A-Info \\
\hline Transmit-receive antenna distance & $5 \mathrm{~m}$ \\
\hline
\end{tabular}

(a) displays one of these reflectarrays design for boresight pointing. The CST simulated results are compared against measured results in section (6). Fig. 9 (b) shows the feed support structure simulated in another CST simulation to discuss sidelobe performance of the measured reflectarrays.

\section{Reflectarray fabrication and measurements}

This section is about the fabrication of phase quantized reflectarray and the anechoic chamber measurement setup used during their measurements.

\subsection{Reflectarray fabrication}

Two 3 bit phase quantized reflectarrays were fabricated using a photolithography printed circuit board $(\mathrm{PCB})$ printing process. Fig. 10 displays the mo-

saics and fabricated reflectarrays on the grounded substrate for pointing their 


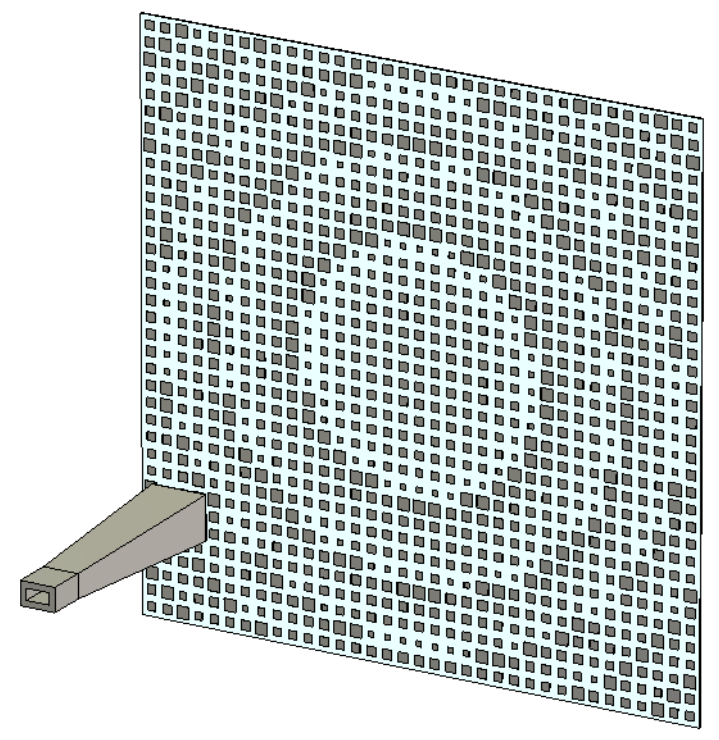

(a) Reflectarray in CST for simulated 1

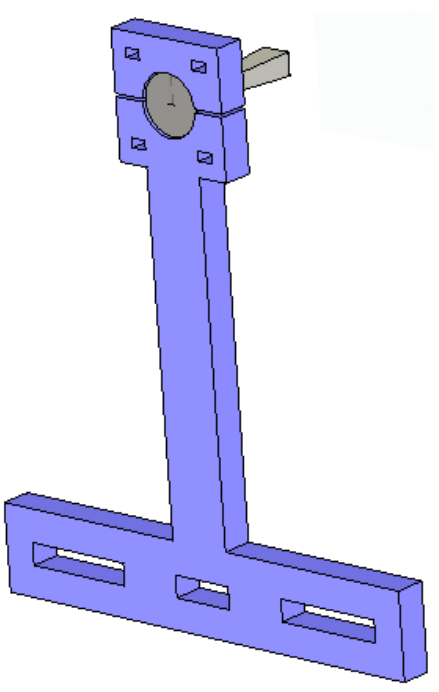

(b) Feed structure in simulated 2

Figure 9: Structure of reflectarray simulated in CST to generate results in Fig. 13 (a) without support structure and feed horn flange to generate results 'Simulated 1', (b) Feed horn flange and feed support included in simulation of reflectarray to generate results 'Simulated 2'.

respective radiated mains beams at $0^{\circ}$ and $55^{\circ}$. Both of the fabricated reflectarrays were individually mounted on rigid back supports to provide mechanical stability.

\subsection{Reflectarray measurement setup}

A simplified diagram of the mm-wave antenna measurement system is shown in Fig. 11 [50].

The millimeter wave antenna measurement system is based on an HP/Agilent 85309A frequency converter, and has a transmitter and a receiver section. Its transmit section is made of a synthesized RF source (Agilent 8350 sweep oscillator), an amplifier (HP 8349B), a mm-wave source module (HP 8355xA), and the transmit antenna. A standard gain horn is used as the transmit antenna. A mm-wave stimulus signal for the receive antenna which is an antenna under test (AUT) is provided by the mm-wave source module and the transmit antenna. 


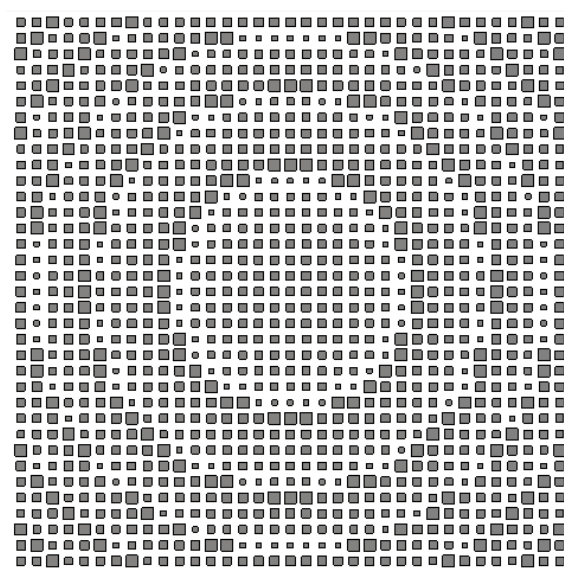

(a) Mosaic for $0^{\circ}$ pointed reflectarray

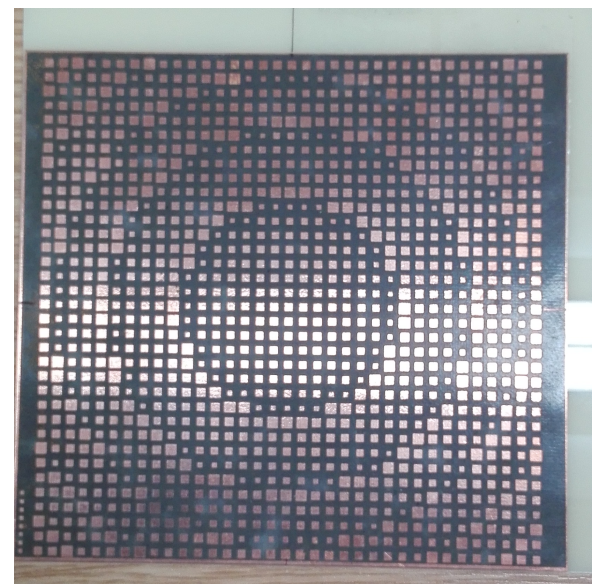

(c) Fabricated reflectarray, pointed at $0^{\circ}$

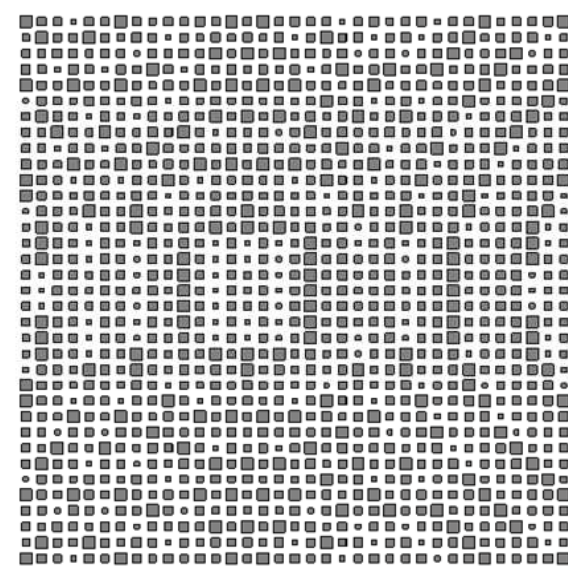

(b) Mosaic for $55^{\circ}$ pointed reflectarray

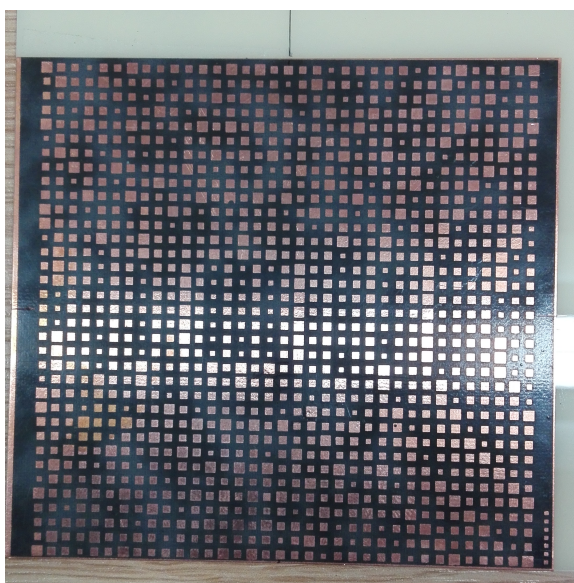

(d) Fabricated reflectarray, pointed at $55^{\circ}$

Figure 10: Two 3 bit phase quantized reflectarrays with their respective radiated main beams pointed at $0^{\circ}$ and $55^{\circ}$. Mosaics used for fabrication are shown in (a, b) while fabricated reflectarrays are shown in (c, d). Both arrays were fabricated on a grounded 10 mil thick RO5880 substrate. Fabricated reflectarrays are mounted on supporting plates to provide mechanical rigidity during measurements.

Through a general purpose interface bus (GPIB interface), the RF source module is controlled by the receiver. HP $8355 x \mathrm{~A}$ mm-wave source module accepts an $\mathrm{RF}$ power input in the range of +17 to $+27 \mathrm{dBm}$. RF source and HP $8349 \mathrm{~B}$ amplifier together provide an output of $+17 \mathrm{dBm}$ for HP $8355 \mathrm{xA}$ mm-wave source module. HP 8355xA mm-wave source module up-converts the RF frequency by a factor of 2 to 6 based on the module used. A source module interface (SMI) 


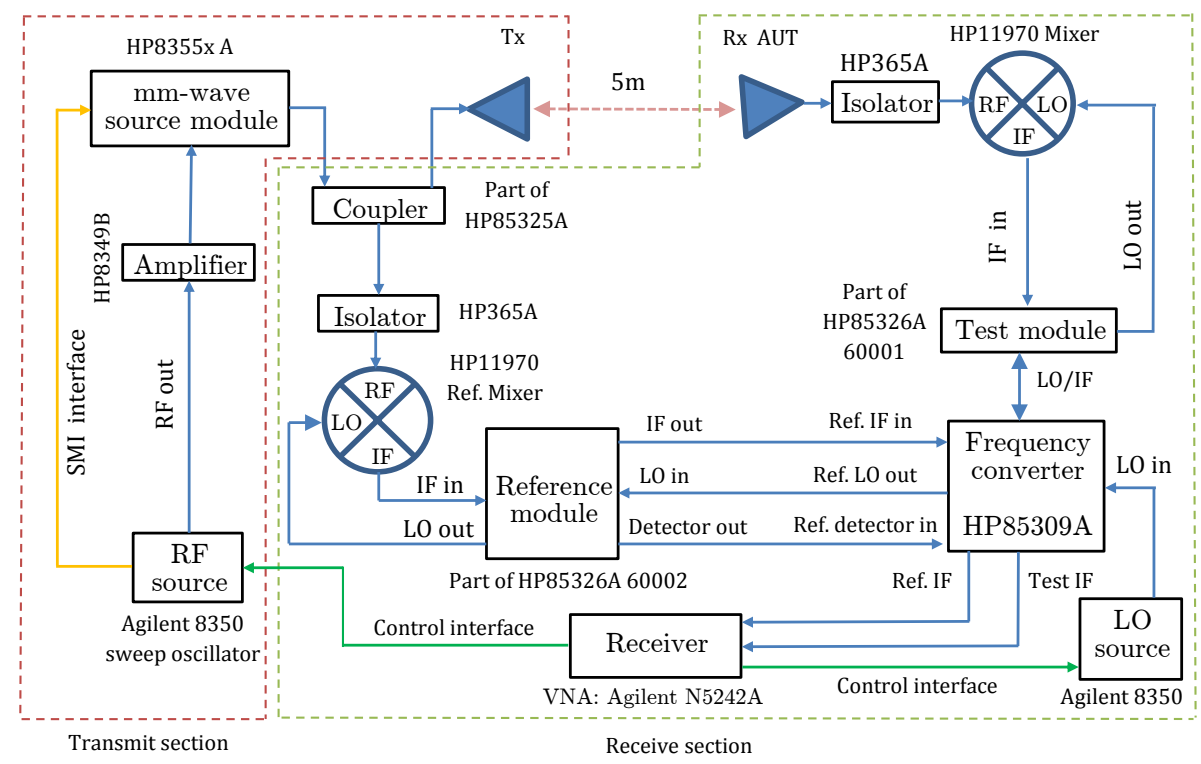

Figure 11: Antenna measurement setup.

290

Due to this interface (with its cables connected), the RF source acknowledges the fact that multiplied frequencies are in use. Therefore, when the receiver asks for frequencies above the RF source's normal range, it responds correctly. Using the STIMULUS controls on the receiver one can change the output power of the mm-wave source module.

The receiver section of the measurement system consists of an AUT or a reference antenna, a directional coupler (shown as a part of HP 85325A: mm-wave interface kit), frequency converter unit, mixer modules (part of HP 85326A: Test-60001 and Ref-60002), mm-wave mixers (HP 11970, two units; one as a ers), and a receiver Agilent N5242A vector network analyzer (VNA). At the input of each mm-wave mixer there is an isolator to improve the impedance matching between the antenna and its mixer. An isolator also blocks mixer harmonic signals from radiating through the antenna. Mixers down-convert the 305 mm-wave signal to a $20 \mathrm{MHz}$ IF frequency for the receiver Agilent N5242A. Both mixers (test and ref.) are identical. However, they are driven by differ- 
ent mixer modules. These mixers operate normally with an LO power in the range of $16 \pm 2 \mathrm{dBm}$. The reference mixer module (60002) measures the LO power it receives, through an internal detector. The output voltage of the LO power detector are fed to HP/Agilent 85309A LO/IF unit which controls the LO power. LO power to the test module (60001) is not measured. The test module receives the same amount of LO power as received by the reference module due to assumed similar electrical distances. Therefore, test and reference LO powers are controlled simultaneously. Test mixer module has a diplexer to allow LO and IF signals to travel through the same cable therefore, permitting a single ring rotary joint for an AUT. The LO signal is provided by an LO source unit. HP/Agilent 85309A LO/IF unit, amplifies and distributes LO signals to mixer modules. HP/Agilent 85309A also controls the LO power levels through an automatic level control circuitry. It receives IF signals from both mixer modules, amplify them and send them to the receiver. At the receiver $20 \mathrm{MHz}$ IF signals are converted to the digital data, processed and displayed. The receiver also controls the frequencies of LO and RF sources through control interfaces. A computer running the data capture software is connected to the receiver to further process and display the measurement data. For more detailed description of the measurement system and its operation one can refer to [50].

Fig. 12(a) displays an assembled reflectarray. It consists of the fabricated reflectarray, the feed horn to spatially illuminate the unit cells of reflectarray, and the mechanical support structure. The mechanical support structure consists of three parts: an interface plat which provides the mounting of the whole assembled reflectarray structure with the antenna positioner, a small tower to hold the feed horn at proper height with respect to the reflectarray aperture, and a base support to hold the feed horn tower. The base support and the feed horn tower are designed to provide the adjustments for reflectarray focal length by sliding the feed horn tower into the prongs of the base support. Fig. 335 12(b) shows the whole assembled structure of reflectarray (antenna under test $(\mathrm{AUT}))$ mounted on the anechoic chamber tower on the receive $(\mathrm{Rx})$ side. The transmit (Tx) side consists of a horn antenna as shown in Fig. 12(c). The three 


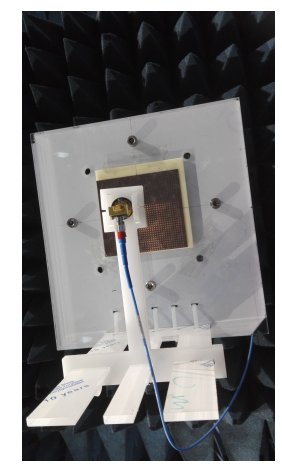

(a) Array test structure

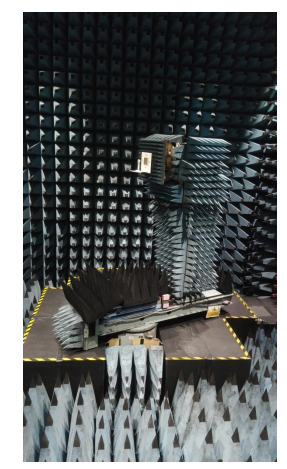

(b) Array on test tower

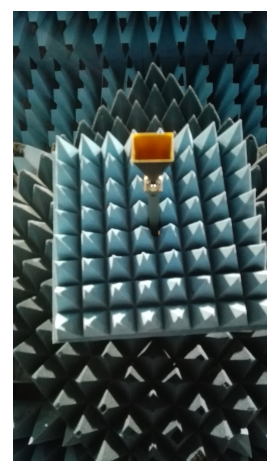

(c) Transmit side horn

Figure 12: Reflectarray measurements in anechoic chamber. (a) Reflectarray and its feed horn mounted on the test structure, (b) Reflectarray and its feed horn with test structure mounted on the antenna test tower in anechoic chamber on the receive side, (c) Transmit side horn antenna.

antenna test method was used to measure the gain of reflectarrays [51. This method uses an additional reference antenna with a known gain in place of the AUT. Therefore, by comparison of the received signal strength the gain of the AUT can be found. The chamber was calibrated using the reference antenna. Similarly, the radiation pattern of reflectarrays was measured by rotating the antenna positioner in azimuth direction at a fixed elevation.

\section{Reflectarray results and discussions}

Two 3 bit phase quantized center fed reflectarrays were simulated in CST, and fabricated for measurements. The radiation beam of one design was pointing at the boresight, while other design was for $55^{\circ}$ pointing. These reflectarrays were measured for the radiation pattern and gain performance in an anechoic chamber measurement setup described in section 5.2. Results from CST simulations and measurements are compared here. CST simulated results are based on $60 \mathrm{GHz}$ center frequency while measured results are based on $61.5 \mathrm{GHz}$ center frequency. CST results were frequency offset by $1.5 \mathrm{GHz}$ to show the comparison with measured results. There is not a practically significant difference in gain from 60 to $61.5 \mathrm{GHz}$ in both simulations and measurements as reflectarrays cover a relatively wider bandwidth. The reasons for this frequency 
shift are the feed horn location (focal point of reflectarrays versus feed horn phase center) and the fabrication process. The results are displayed in Fig. 13. Fig. 13 (a) and (c) show normalized radiation patterns at $61.5 \mathrm{GHz}$ for both arrays, while (b) and (d) show gain-bandwidth response along with the array pointing angle response. In each case the CST simulated results are plotted to show a comparison. Each radiation pattern curve is normalized to its own maximum value. A very good overlap of mainlobes, beam pointing directions, and gain-bandwidths can be observed in these figures. A key observation here is the gain-bandwidth decreases as the beam is pointed away from the antenna boresight. The measured cross polarization isolation performance was better than $38 \mathrm{~dB}$ in both cases. Similarly, the measured reflection coefficient at the feed horn port for the impedance matching purpose was better than $-20 \mathrm{~dB}$. The difference in sidelobes' performance is discussed as follows.

The curve marked 'Simulated 1' in Fig. 13 (a) is the CST simulation of reflectarray with feed horn only as shown in Fig. 9 (a). A very good sidelobe performance can be observed in this case. During measurements we used the support structure as shown in Fig. 12 (a) which is made of acrylic sheets $\left(\epsilon_{\mathrm{r}} \approx 3\right)$. To simulate the whole support structure in CST was not possible due to huge computation requirements. We consider that the feed support 375 structure as shown in Fig. 9 (b) is the main contributor to an increase in sidelobe levels. Therefore, the feed support structure and its flange were modeled in CST simulations. The effect of flange was to reduce the gain slightly $(0.4 \mathrm{~dB})$ and increase sidelobes by a few $\mathrm{dBs}(2 \mathrm{~dB})$. When the feed support is included in simulations, it took around 105 hours of simulation time on an high performance computation cluster to generate results marked as 'Simulated 2' in Fig. 13 (a). One can observe that the sidelobes are significantly higher than those shown by 'Simulated 1'. Because the whole support structure was not simulated, therefore the measured sidelobes' performace do not match very well with simulated cases. However, a trend of rising sidelobes due to a non-air support is visible. Rohacell support would perform better in this case instead of the acrylic support and one would be able to achieve a sidelobe performance close to the 'Simulated 1' case. 


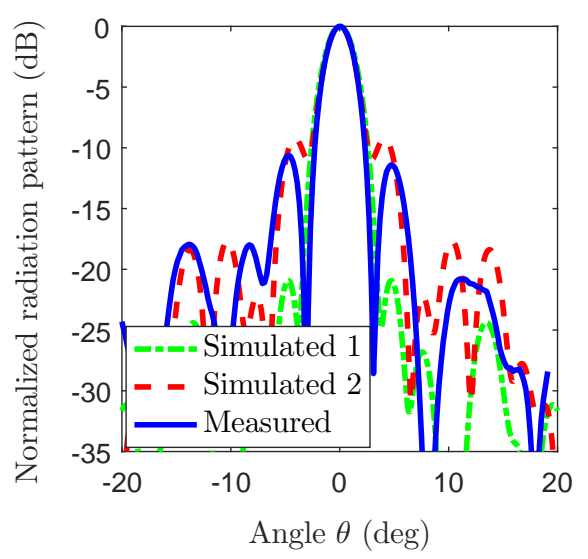

(a) Radiation pattern for $0^{\circ}$ pointing

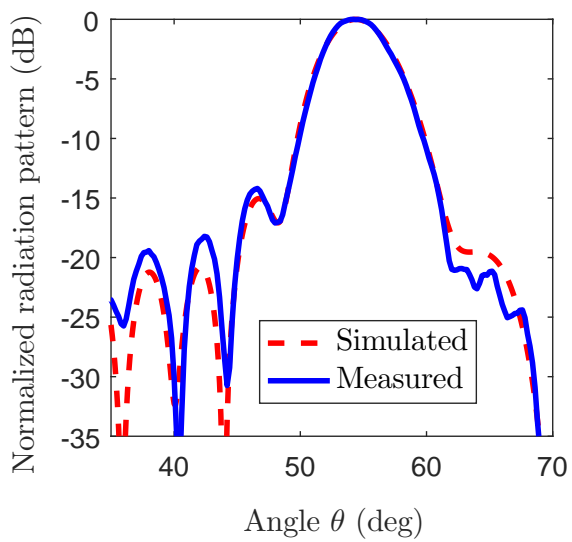

(c) Radiation pattern for $54.2^{\circ}$ pointing

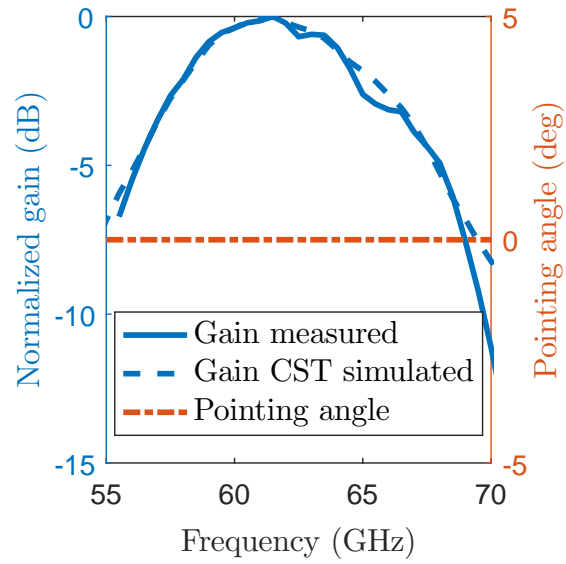

(b) Bandwidth response at $0^{\circ}$

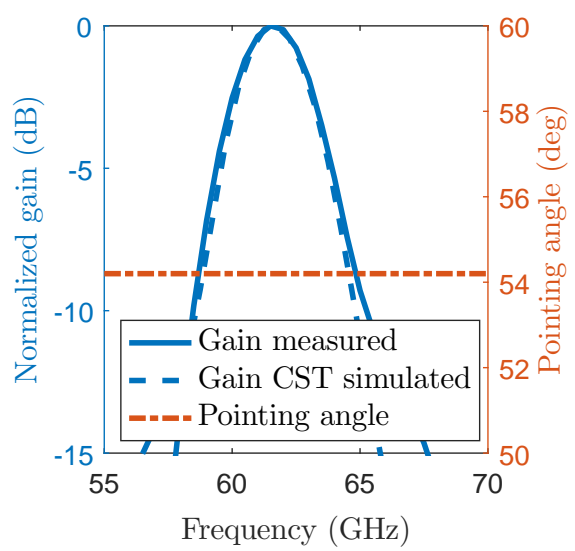

(d) Bandwidth response at $54.2^{\circ}$

Figure 13: The response of two $\mathrm{V}$ band 3.0 bit phase quantized reflectarrays designed to point their respective main beams at $0^{\circ}$ and $55^{\circ}$. A comparison of measured results with CST simulated results for radiation pattern and bandwidth at their respective pointing angles.

In Fig. 13 (c), because the main beam is pointing off boresight therefore, the effects of blockage due to the feed horn and its support are significantly reduced. The curve marked 'Simulated' displays the CST simulated results without considering the feed horn flange and any support structures for this $55^{\circ}$ reflectarray design just like the case of Fig. 9 (a) with reflectarray replaced with the one pointing at $55^{\circ}$. Due to a significant reduction in blockage, the 
sidelobes match much better than those stated for $0^{\circ}$ pointing reflectarray. It should be noted that the reflectaray designed for $55^{\circ}$ beam pointing achieved beam pointing angle of $54.2^{\circ}$ which is the same angle one would achieve through the array theory based analysis of reflectarrays as shown by authors in [49].

Table 2: A comparison of CST simulated and measured results for phase quantized reflectarrays at fixed beam pointing angles. $(35 \times 35$ elements, inter-element spacing $=2.7 \mathrm{~mm}, F$ $=70 \mathrm{~mm}, L=W=94.5 \mathrm{~mm}$, feed horn model LB-15-10-A from A-Info, frequency for CST results $=60 \mathrm{GHz}$, frequency for test results $=61.5 \mathrm{GHz}$ )

\begin{tabular}{|l|l|l|l|l|l|l|}
\hline $\begin{array}{l}\text { Bit: } \\
\text { angle }\end{array}$ & $\begin{array}{l}\text { Gain } \\
\mathbf{C S T} \\
\mathbf{( d B i})\end{array}$ & $\begin{array}{l}\text { Beam- } \\
\text { width } \\
\mathbf{C S T}\end{array}$ & $\begin{array}{l}\text { Bandwidth } \\
\mathbf{C S T}(\mathbf{G H z})\end{array}$ & $\begin{array}{l}\text { Gain } \\
\text { test } \\
\mathbf{( d B i})\end{array}$ & $\begin{array}{l}\text { Beam- } \\
\text { width } \\
\text { test }\end{array}$ & $\begin{array}{l}\text { Bandwidth } \\
\text { test } \mathbf{( G H z )}\end{array}$ \\
\hline $3: 0^{\circ}$ & 34.55 & $2.9^{\circ}$ & 8.92 & 33.26 & $2.75^{\circ}$ & 9.37 \\
\hline $3:$ & 30.7 & $5.5^{\circ}$ & 3.26 & 31.39 & $5.06^{\circ}$ & 3.51 \\
$54.2^{\circ}$ & & & & & & \\
\hline
\end{tabular}

Table 2 lists the major performance parameters of these 3 bit phase quantized reflectarrays. In Table 2 'Bit: angle' = number of phase quantization bit of the reflectarray and the achieved pointing angle, 'Gain CST' = gain achieved in CST Microwave Studio FW-EM simulations using time domain (T) solver, 'Bandwidth $\mathrm{CST}^{\prime}=$ achieved $3 \mathrm{~dB}$ gain bandwidth in CST simulations, 'Gain test' $=$ measured gain using 3 antenna test method, 'Bandwidth test' $=3 \mathrm{~dB}$ gain bandwidth from measurements, 'Beamwidth CST' $=3 \mathrm{~dB}$ beamwidth achieved in CST simulations, 'Beamwidth test' = is the measured $3 \mathrm{~dB}$ beamwidth.

A comparison of this work with the reflectarray measured results based on literature is given in Table 3. One would be cautious while comparing these parameters, as each reflectarray design is different and also the unit cell design vary significantly, which makes such a comparison hard. Majority of designs are based on lower frequency bands which are much more robust to fabrication tolerances in comparison to lower frequency bands. A fair comparison with this work is the reflectarray at $77 \mathrm{GHz}$ presented in 20. One obvious fact is this work proposes phase quantization in passive reflectarrays to ease imple- 
mentation at mm-waves which is not considered in other works. Another fact is this work managed to achieve the efficiency comparable to parabolic reflector antennas although while implementing phase quantization. This highlights the importance of unit cell parametric studies using analytical tool presented in this work for an optimum design.

Table 3: A comparison with existing literature

\begin{tabular}{|l|l|l|l|l|l|}
\hline Parameter & This work & {$[\mathbf{5 2}]$} & {$[\mathbf{5 3}]$} & {$[\mathbf{2 0}]$} & {$[\mathbf{5 4}]$} \\
\hline Frequency $(\mathrm{GHz})$ & 60 & 30 & 12.5 & 77 & 11.5 \\
\hline Size $(\lambda)$ gain & 19 & 6 & 20 & 39 & 13 \\
\hline $\begin{array}{l}\text { Measured } \\
(\mathrm{dBi})\end{array}$ & 21.7 & 26.8 & 36 & 24 \\
\hline $\mathrm{dB}$, Bandwidth (\%) & $3,15.6$ & - & - & $1,2.5$ & $3,47.8$ \\
\hline Efficiency (\%) & 60 & 42 & 23 & 27 & 50 \\
\hline Cross pol isolation & 38 & 21 & - & - & 20 \\
\hline Phase qunatization & Yes & No & Yes & No & No \\
\hline
\end{tabular}

\section{Conclusion}

We presented a numerically efficient closed form analytical solution for a single layer rectangular/square printed mm-wave reflectarray unit cells including the effects of fringing fields, finite metal conductivity, metal surface roughness, and surface waves. The analytical technique was compared with CST simulations and a strong agreement was found. To ease the implementation of mmwave reflectarrays, the phase quantization was proposed. Using the presented analytical technique two 3 bit phase quantized reflectarrays were designed, fabricated and measured. Their measurement results were compared with the CST simulations and a very good agreement of results was observed. Therefore, a reliable framework to design high gain reflectarrays is established. These findings are particularly important for small satellites where accommodation space is 

inter-satellite links.

\section{Acknowledgements}

Authors are thankful to the fabrication team at University of Surrey technical support unit. Many thanks to the measurements team at NPL for their support. This research was jointly sponsored by the Surrey Space Centre, National Physical Laboratory [grant number KB4545], and National Measurement Office U.K. [grant numbers 116774, 119575].

\section{References}

[1] S. Zihir, O. D. Gurbuz, A. Karroy, S. Raman, G. M. Rebeiz, A 60 GHz 64element wafer-scale phased-array with full-reticle design, in: IEEE MTT-S Int. Microw. Symp., 2015, pp. 1-3. doi:10.1109/MWSYM.2015.7167089.

[2] S. Zihir, O. D. Gurbuz, A. Kar-Roy, S. Raman, G. M. Rebeiz, 60-GHz 64and 256-elements wafer-scale phased-array transmitters using full-reticle and subreticle stitching techniques, IEEE Trans. on Microw. Theory and Tech. 64 (12) (2016) 4701-4719. doi:10.1109/TMTT.2016.2623948

[3] H. Kamoda, T. Iwasaki, J. Tsumochi, T. Kuki, 60-GHz electrically reconfigurable reflectarray using pin diode, in: IEEE MTT-S Int. Microw. Symp., 2009, pp. 1177-1180.

[4] H. Kamoda, T. Iwasaki, J. Tsumochi, T. Kuki, O. Hashimoto, 60-GHz electronically reconfigurable large reflectarray using single-bit phase shifters, IEEE Trans. on Antennas and Propag. 59 (7) (2011) 2524-2531.

[5] J. Huang, Reflectarray antenna, Wiley Online Library, 2008.

[6] S. V. Hum, Reflectarrays, in: Aperture Antennas for Millimeter and SubMillimeter Wave Applications, Springer, 2018, pp. 143-189.

[7] P. Nayeri, F. Yang, A. Z. Elsherbeni, Reflectarray Antennas: Theory, Designs, and Applications, Wiley Online Library, 2018.

[8] J. Huang, A. Feria, A 1-m x-band inflatable reflectarray antenna, Microwave and Optical Technology Letters 20 (2) (1999) 97-99.

[9] Y. MiYazaki, Deployable techniques for small satellites, Proceedings of the IEEE 106 (3) (2018) 471-483. doi:10.1109/JPROC.2018.2799608. 
[10] Z.-Q. Liu, H. Qiu, X. Li, S.-L. Yang, Review of large spacecraft deployable membrane antenna structures, Chinese Journal of Mechanical Engineering 30 (6) (2017) 1447. doi:10.1007/s10033-017-0198-x.

[11] L. Zou, M. Cryan, M. Klemm, Phase change material based tunable reflectarray for free-space optical inter/intra chip interconnects, Opt. Express 22 (20) (2014) 24142-24148. doi:10.1364/OE.22.024142.

(1) URL http://www.opticsexpress.org/abstract.cfm?URI= oe-22-20-24142

[12] Y. Yifat, M. Eitan, Z. Iluz, Y. Hanein, A. Boag, J. Scheuer, Highly efficient 470 and broadband wide-angle holography using patch-dipole nanoantenna reflectarrays, Nano Letters 14 (5) (2014) 2485-2490, pMID: 24646057. arXiv:https://doi.org/10.1021/nl5001696, doi:10.1021/nl5001696. URL https://doi.org/10.1021/n15001696

[13] V. Lapanik, G. Sasnouski, S. Timofeev, E. Shepeleva, G. Ev475 a tyushkin, W. Haase, New highly anisotropic liquid crystal materials for high-frequency applications, Liquid Crystals 45 (8) (2018) 1242-

11 1249. arXiv:https://doi.org/10.1080/02678292.2018.1427810, doi: 10.1080/02678292.2018.1427810.

URL https://doi.org/10.1080/02678292.2018.1427810

${ }_{480}$ [14] S. R. Biswas, C. E. Gutiérrez, A. Nemilentsau, I.-H. Lee, S.-H. Oh, a P. Avouris, T. Low, Tunable graphene metasurface reflectarray for cloak1 ing, illusion, and focusing, Phys. Rev. Applied 9 (2018) 034021. doi: 10.1103/PhysRevApplied.9.034021.

URL https://link.aps.org/doi/10.1103/PhysRevApplied.9.034021

485 [15] T. Debogovic, J. Perruisseau-Carrier, Dual-polarized low loss reflectarray cells with mems-based dynamic phase control, in: Antennas and Propagation (EuCAP), 2015 9th European Conference on, IEEE, 2015, pp. 1-5.

[16] S. V. Hum, J. Perruisseau-Carrier, Reconfigurable reflectarrays and array lenses for dynamic antenna beam control: A review, IEEE Trans. on Antennas and Propag. 62 (1) (2014) 183-198. doi:10.1109/TAP.2013.2287296

[17] M. Zhou, O. Borries, E. Jørgensen, Design and optimization of a single-layer planar transmit-receive contoured beam reflectarray with enhanced performance, IEEE Transactions on Antennas and Propagation 63 (4) (2015) 1247-1254. doi:10.1109/TAP.2014.2365039.

495 [18] D. Pozar, Bandwidth of reflectarrays, IET Electronics Letters 39 (21) (2003) 1490-1491.

[19] D. Pozar, T. Metzler, Analysis of a reflectarray antenna using microstrip patches of variable size, IET Electronics Letters 29 (8) (1993) 657-658. 
[20] D. M. Pozar, S. D. Targonski, H. Syrigos, Design of millimeter wave microstrip reflectarrays, IEEE trans. on antennas and propag. 45 (2) (1997) 287-296. doi:10.1109/8.560348.

[21] H. Rajagopalan, Y. Rahmat-Samii, On the reflection characteristics of a reflectarray element with low-loss and high-loss substrates, IEEE Antennas and Propag. Mag. 52 (4) (2010) 73-89.

[22] M. Y. Zhao, G. Q. Zhang, X. Lei, J. M. Wu, J. Y. Shang, Design of new single-layer multiple-resonance broadband circularly polarized reflectarrays, IEEE Antennas and Wireless Propag. Letters 12 (2013) 356-359.

[23] S. Nesil, F. Güneş, U. Özkaya, Phase characterization of a reflectarray unit cell with minkowski shape radiating element using multilayer perceptron neural network, in: 7th Int. Conf. on Electrical and Electronics Engineering (ELECO), IEEE, 2011, pp. II-219.

[24] H. Hasani, C. Peixeiro, A. K. Skrivervik, J. Perruisseau-Carrier, Singlelayer quad-band printed reflectarray antenna with dual linear polarization, IEEE Trans. on Antennas and Propag. 63 (12) (2015) 5522-5528.

[25] X. Xia, Q. Wu, H. Wang, C. Yu, W. Hong, Wideband microstrip reflectarray using dual-resonance unit cell, in: Asia-Pacific Microw. Conf.(APMC), Vol. 2, IEEE, 2015, pp. 1-3.

[26] M. M. Tahseen, A. A. Kishk, High efficiency Ka-band single layer air vias reflectarray: Design and analysis, in: IEEE Int. Symp. on Antennas and Propag. USNC/URSI National Radio Science Meeting, IEEE, 2015, pp. 2129-2130.

[27] H. Nematollahi, J.-J. Laurin, J. Page, J. A. Encinar, Design of broadband transmitarray unit cells with comparative study of different numbers of layers, IEEE Trans. on Antennas and Propag. 63 (4) (2015) 1473-1481.

[28] Z. Dai, Y. Shang, F. Yao, X. Xuan, Analysis and design of beam-scanning reflectarray with circular polarization., in: PIERS Proc., 2014.

[29] M. K. Arshad, F. Tahir, Optimum microstrip reflectarray unit cell design for wide-band operation, in: 11th Int. Conf. on Frontiers of Information Technology (FIT), IEEE, 2013, pp. 150-153.

[30] H. A. Haus, Waves and fields in optoelectronics, Prentice-Hall, 1984.

[31] A. Z. Elsherbeni, P. Nayeri, F. Yang, Reflectarray antennas for space applications, in: IEEE Int. Conf. on Ultra-Wideband (ICUWB), IEEE, 2012, pp. $362-365$.

[32] F. Yang, P. Nayeri, A. Z. Elsherbeni, Recent advances in beam-scanning reflectarray antennas, in: XXXIth URSI General Assembly and Scientific Symp. (URSI GASS), IEEE, 2014, pp. 1-4. 
[33] P. Hannan, M. Balfour, Simulation of a phased-array antenna in waveguide, IEEE trans. on Antennas and Propag. 13 (3) (1965) 342-353.

[34] D. M. Pozar, Microwave engineering, John Wiley \& Sons, 2009.

540 [35] K. Carver, J. Mink, Microstrip antenna technology, IEEE trans. on antennas and propag. 29 (1) (1981) 2-24.

[36] Rogers Corporation, Copper foils for high frequency circuit materials, https://www.rogerscorp.com/documents/749/acs/ Copper-Foils-for-High-Frequency-Circuit-Materials.pdf $\quad$ (accessed 28 March 2018).

[37] H. Braunisch, X. Gu, A. Camacho-Bragado, L. Tsang, Off-chip roughmetal-surface propagation loss modeling and correlation with measurements, in: Proc. of 57th Electronic Components and Technology Conf. (ECTC'07), IEEE, 2007, pp. 785-791.

[38] S. Hinaga, M. Koledintseva, P. Anmula, J. Drewniak, Effect of conductor surface roughness upon measured loss and extracted values of PCB laminate material dissipation factor, in: Proc. Technol. Conf. IPC Expo/APEX, 2009, pp. S20-2.

[39] D. M. Pozar, Rigorous closed-form expressions for the surface wave loss of printed antennas, IET Electronics Letters 26 (13) (1990) 954-956.

[40] G. Ahmad, C. Underwood, T. Brown, T. Loh, Role of surface waves in improving the reflection properties of a millimetre wave reflectarray unit cell, in: Proc. of Loughborough Antennas and Propag. Conf. (LAPC), The Institution of Engineering and Technology, 2017.

[41] B. Constantine A, Antenna theory: analysis and design, 3rd Edition, John wiley \& sons, 2005.

[42] M. Kara, Formulas for the computation of the physical properties of rectangular microstrip antenna elements with various substrate thicknesses, Microw. and Optical Tech. Letters 12 (4) (1996) 234-239.

[43] R. Bancroft, Microstrip and printed antenna design, The Institution of Engineering and Technology, 2009.

[44] H. A. Wheeler, Transmission-line properties of parallel strips separated by a dielectric sheet, IEEE Trans. on Microw. Theory and Techniques 13 (2) (1965) 172-185.

570 [45] E. O. Hammerstad, Equations for microstrip circuit design, in: 5th European Microw. Conf., IEEE, 1975, pp. 268-272. 
[46] Rogers Corporation, Width and effective dielectric constant equations for design of microstrip transmission lines, https://www.rogerscorp.com/documents/783/acs/

575 Design-Data-for-Microstrip-Transmission-Lines-of-TMM-Laminates. pdf (accesseed 23 March 2016).

[47] W. Richards, Y. Lo, P. Simon, D. Harrison, Theory and applications for microstrip antennas, in: Proc. Workshop on Printed Circuit Antenna Technol., 1979, pp. 8-1.

[48] R. Collier, P. White, Surface waves in microstrip circuits, in: 6th European Microw. Conf., IEEE, 1976, pp. 632-636.

[49] G. Ahmad, T. W. Brown, C. I. Underwood, T. H. Loh, How coarse is too coarse in electrically large reflectarray smart antennas?, in: Int. Workshop on Electromagnetics Applications and Student Innovation Competition (iWEM), IEEE, 2017, pp. 135-137.

[50] Agilent Technologies 85325A R/Q/U/V/W millimeter subsystem: Operating and service manual, https://literature.cdn.keysight.com/ litweb/pdf/85325-90061.pdf?id=1000002822-1: epsg:man (accessed 28 March 2018).

590 [51] IEEE standard test procedures for antennas (1979).

[52] Q. Luo, S. Gao, C. Zhang, D. Zhou, T. Chaloun, W. Menzel, V. Ziegler, M. Sobhy, Design and analysis of a reflectarray using slot antenna elements for ka-band satcom, IEEE Trans. on Antennas and Propag. 63 (4) (2015) 1365-1374. doi:10.1109/TAP.2015.2401393.

[53] H. Yang, F. Yang, S. Xu, M. Li, X. Cao, J. Gao, Y. Zheng, A study of phase quantization effects for reconfigurable reflectarray antennas, IEEE

n. Antennas and Wireless Propag. Letters 16 (2017) 302-305. doi:10.1109/ LAWP.2016.2574118

[54] L. Zhang, S. Gao, Q. Luo, W. Li, Y. He, Q. Li, Single-layer wideband circularly polarized high-efficiency reflectarray for satellite communications, IEEE Trans. on Antennas and Propag. 65 (9) (2017) 4529-4538. doi:10.1109/TAP.2017.2722824. 